\title{
BMJ Open Study protocol for a multicentre longitudinal mixed methods study to explore the Outcomes of ChildrEn and fAmilies in the first year after paediatric Intensive Care: the OCEANIC study
}

To cite: Manning JC, Latour JM, Curley MA.Q, et al. Study protocol for a multicentre longitudinal mixed methods study to explore the Outcomes of ChildrEn and fAmilies in the first year after paediatric Intensive Care: the OCEANIC study. BMJ Open 2020;10:e038974. doi:10.1136/ bmjopen-2020-038974

- Prepublication history and additional material for this paper are available online. To view these files, please visit the journal online (http://dx.doi. org/10.1136/bmjopen-2020038974).

Received 30 March 2020 Revised 16 April 2020 Accepted 21 April 2020

D) Check for updates

(c) Author(s) (or their employer(s)) 2020. Re-use permitted under CC BY. Published by BMJ.

For numbered affiliations see end of article.

\section{Correspondence to} Dr Joseph C Manning; joseph.manning@nottingham. ac.uk

\section{ABSTRACT}

Introduction Annually in the UK, 20000 children become very ill or injured and need specialist care within a paediatric intensive care unit (PICU). Most children survive. However, some children and their families may experience problems after they have left the PICU including physical, functional and/or emotional problems. It is unknown which children and families experience such problems, when these occur or what causes them. The aim of this mixed-method longitudinal cohort study is to understand the physical, functional, emotional and social impact of children surviving PICU (aged: 1 month-17 years), their parents and siblings, during the first year after a PICU admission.

Methods and analysis A quantitative study involving 300 child survivors of PICU; 300 parents; and 150-300 siblings will collect data (using self-completion questionnaires) at baseline, PICU discharge, 1, 3, 6 and 12 months postPICU discharge. Questionnaires will comprise validated and reliable instruments. Demographic data, PICU admission and treatment data, health-related quality of life, functional status, strengths and difficulties behaviour and post-traumatic stress symptoms will be collected from the child. Parent and sibling data will be collected on the impact of paediatric health conditions on the family's functioning capabilities, levels of anxiety and social impact of the child's PICU admission. Data will be analysed using descriptive and inferential statistics. Concurrently, an embedded qualitative study involving semistructured interviews with 24 enrolled families at 3 months and 9 months post-PICU discharge will be undertaken. Framework analysis will be used to analyse the qualitative data.

Ethics and dissemination The study has received ethical approval from the National Health Services Research Ethics Committee (Ref: 19/WM/0290) and full governance clearance. This will be the first UK study to comprehensively investigate physical, functional, emotional and social consequences of PICU survival in the first-year postdischarge.

\section{Strengths and limitations of this study}

- The Outcomes of ChildrEn and fAmilies in the first year after paediatric Intensive Care (OCEANIC) study will be the first multisite, comprehensive study conducted in the UK to investigate the physical, functional, emotional and social consequences of paediatric intensive care unit (PICU) survival in the first-year postdischarge.

- Our longitudinal study design will allow us to look at changes over time in the same patient/family, providing insights into the temporal sequence of changes that may occur as a result of childhood critical illness/injury.

- The qualitative study (interviews with children, parents and siblings) will be analysed in conjunction with quantitative data allowing a fuller understanding of physical, functional, emotional and social consequences of being on PICU and any outstanding needs.

- The primary limitation of this study is loss to followup and missing data points that would challenge the internal validity of reported results from the OCEANIC study.

Clinical Trials Registration Number: ISRCTN28072812 [Preresults]

\section{INTRODUCTION}

In the UK annually, approximately 20000 children (aged 0-18 years) experience a critical illness, requiring paediatric intensive care unit (PICU) treatment and care. ${ }^{1}$ Despite increasing demand on paediatric critical care services, PICU survival has increased substantially over the past three decades, rendering mortality alone an insufficient metric for outcomes assessment post-PICU discharge. ${ }^{2}$ 
Over $96 \%$ of children admitted to PICU survive. ${ }^{1}$ However, the decline in mortality has been accompanied by a concomitant increase in morbidity. ${ }^{3}$ Evidence is building which portrays a cohort of PICU survivors who are physically deconditioned, cognitively impaired and emotionally distraught. The emotional and social health of the PICU survivor's parents and siblings may also be affected. $^{45}$

Two systematic reviews reported that approximately $25 \%$ of critically ill children exhibited negative psychological and behavioural responses within the first-year postdischarge. ${ }^{6}$ Similar themes were identified in a systematic review of qualitative studies examining the psychosocial impact of PICU hospitalisation on children, ${ }^{8}$ lending support to the importance in identifying children suffering from psychological sequelae. Given that psychological well-being is shaped by multiple factors, alterations in the child's sense of self and interpersonal relationships, as well as ongoing worries and fears about hospitalisation, have the potential to affect recovery during the early postdischarge period, and during critical periods of growth and development. Health-related quality of life studies identify deterioration in the emotional well-being of $20 \%-30 \%$ of children up to 1-year post-PICU discharge, ${ }^{67}$ suggesting a sustained effect.

The impact of a child's critical illness on family members may be profound as they, too, can experience psychosocial sequelae. $^{5} 9$ Family members' responses may, in turn, influence the outcomes of child survivors following paediatric critical illness. Furthermore, there is evidence that critical illness impacts a family's social functioning in relation to reintegration with peers, the child and family's social capital and the economic impact of unemployment on families when a caregiver has to relinquish work responsibilities to care for a child. ${ }^{10}$ However, the interplay among the child, their parent and siblings' outcomes, caregiver roles and family needs, and how these change over time are largely absent in the literature. Globally $^{11-13}$ and in the $\mathrm{UK},{ }^{14}{ }^{15}$ researchers, clinicians and patients and their families have recognised understanding and supporting adult survivors of intensive care is both a research and clinical priority. Both patient and public consultation, and a national survey, conducted with the PICU community (including children, their families, service providers and commissioners) confirms that understanding and optimising the outcomes of children and their families are also a research priority for childhood survivors of PICU. ${ }^{16} 17$

\section{METHODS AND ANALYSIS}

\section{Study purpose and objectives}

The purpose of the Outcomes of ChildrEn and fAmilies in the first year after paediatric Intensive Care (OCEANIC) study is to explore child PICU survivors' health outcomes and family impact over 1-year post-PICU discharge.

OCEANIC has four specific objectives:

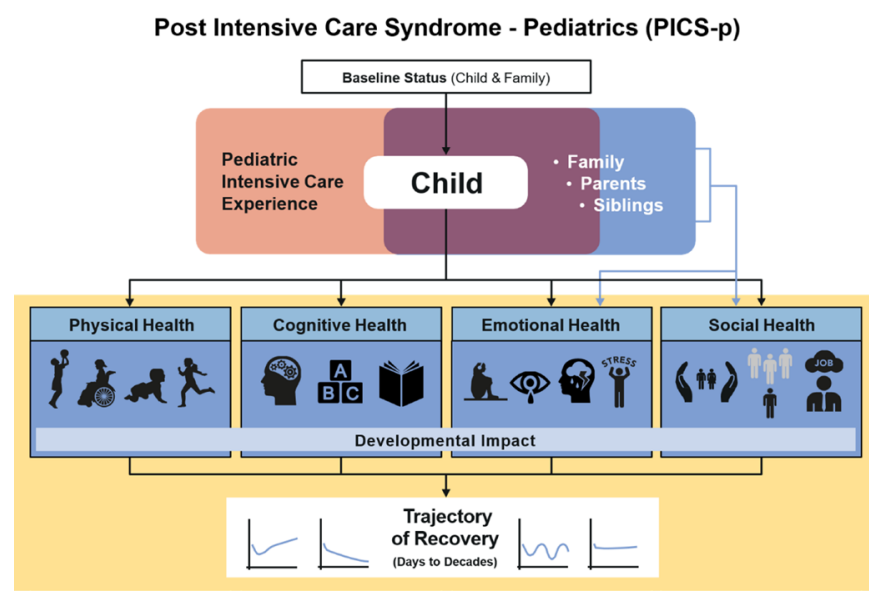

Figure 1 Postintensive care syndrome in paediatrics (PICS-p) framework. ${ }^{19}$

1. To describe the physical, cognitive, emotional and social health outcomes and trajectory of recovery in children post-PICU discharge.

2. To determine the baseline and PICU factors associated with impaired outcomes.

3. To explore the longitudinal emotional and social health outcomes of parents and siblings.

4. To ascertain the care and support needs of children and their parents and siblings.

\section{Theoretical framework}

Based on a state-of-the-science review of postdischarge outcomes in paediatric critical care, ${ }^{18}$ a conceptual framework describing the constellation of potential physical, cognitive, emotional and social health effects that may be uniquely experienced by children and families who survive paediatric critical illness has been proposed (figure 1). ${ }^{19}$ This framework incorporates the importance of pre-existing health status, sociodemographic data, physiological maturation and psychosocial development on the trajectory of health recovery over a child's lifetime. Additionally, the framework recognises that the interdependence of the child and family is central to understanding the long-term multidimensional sequelae of paediatric critical illness. This framework provides a roadmap for understanding longitudinal outcomes; the proposed study will organise data collection using this framework.

This embedded mixed-method study involves two linked work packages (overview presented in figure 2). The first work package will be a quantitative study involving 300 child survivors of critical illness, 300 parents and 150-300 siblings. The second work package will be a qualitative interview study of 2 cohorts of 12 families, at 3 and 9 months post-PICU discharge. Mixing will occur through the sampling and selection of participants for the embedded qualitative study from those enrolled in the quantitative study, as well as in the framework analysis. 


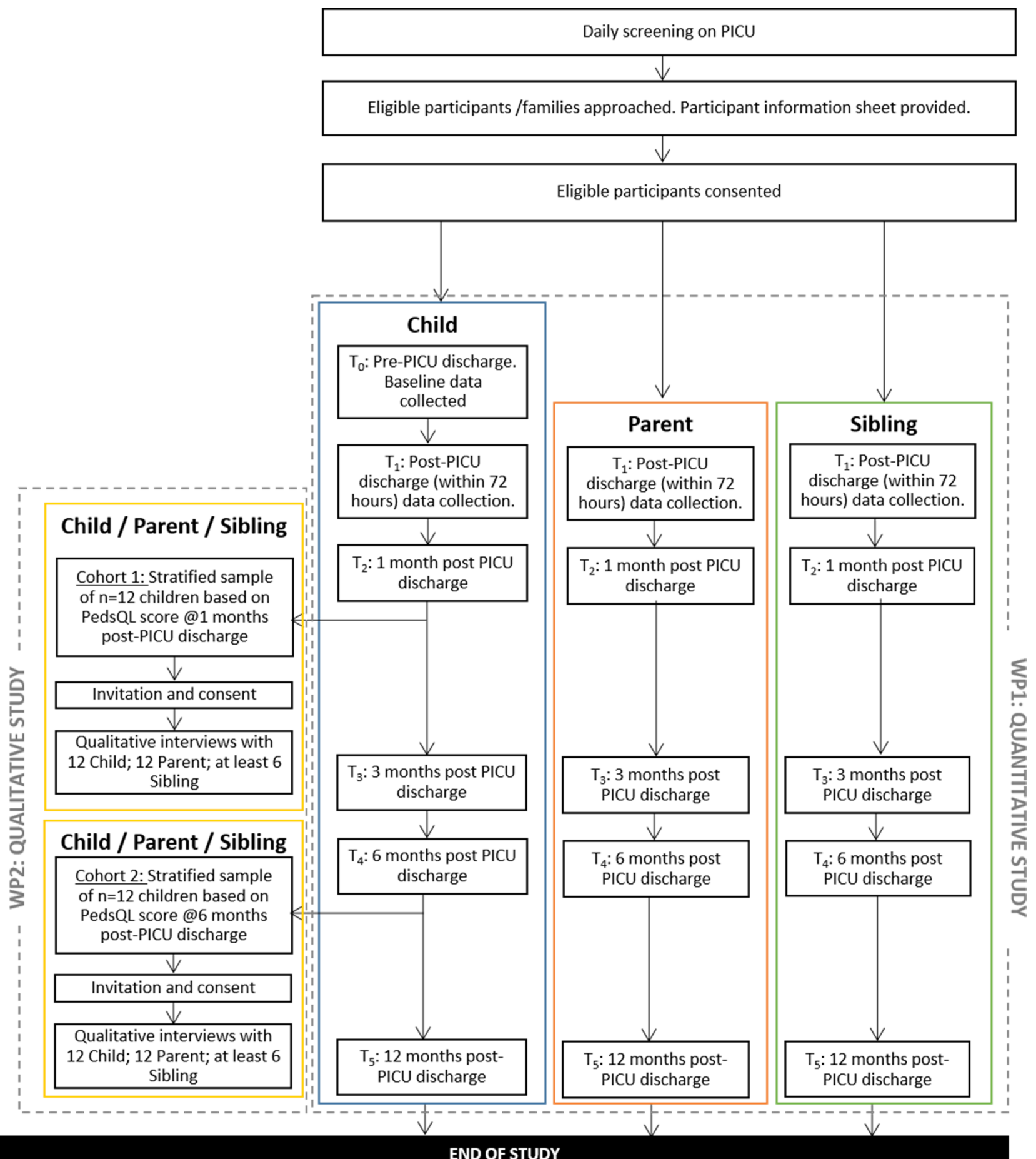

Figure 2 Overview of linked work packages of the Outcomes of ChildrEn and fAmilies in the first year after paediatric Intensive Care study. PedsQL, Pediatric Quality of Life Inventory; PICU, paediatric intensive care unit.

\section{Quantitative study}

Data regarding the PICU admission of each child participant will be downloaded from the Paediatric Intensive Care Audit Network (PICANet) database, a secure and confidential high-quality clinical database of paediatric intensive care activity in the UK and Ireland. Data extracted will include: demographic and socioeconomic data, prePICU health status and acute illness data (PICU admission and discharge diagnoses; comorbidities; operations and invasive procedures performed; type of admission (planned/unplanned); PICU and hospital length of stay (LOS), duration of mechanical ventilation, high-frequency oscillatory ventilation, extracorporeal membrane oxygenation, renal replacement therapy and vasopressor/ inotropic support; sedative medications and days of exposure). Outcome data will also be collected from each child (or proxy), their parent and sibling (if appropriate) prospectively over the first-year post-PICU discharge. 


\section{Study measures}

Currently, there are no standardised or agreed set of outcome measures for research with the PICU patient population. Therefore, the outcome measures used in this study were selected for their validity, reliability, ease of use, availability in electronic versions and previous use with the population under investigation. Furthermore, the focus and selection of these measures were informed by the postintensive care syndrome in paediatrics framework, contemporary literature and consultation with patients, public and PICU clinicians. In line with feedback from patient and public involvement (PPI) consultations, outcomes will be collected at six time points: baseline status (pre-PICU discharge); at PICU discharge; 1, 3, 6 and 12 months post-PICU discharge. The outcomes measured and time points are outlined in table 1 .

Data collection measures, versions and report format according to age and study participant (child PICU survivor, parent/legal guardian or sibling) are reported in table 2. A brief overview of the measures is provided in online supplementary file 1 .

Child related measures include:

- Pediatric Quality of Life Inventory (PedsQL 4.0) Generic Core Scales (2-17 years) and Infant Scales (1-23 months) -Acute Version. ${ }^{30-29}$

- PedsQL Multidimensional Fatigue Scale (2-17 years)-Acute Version. ${ }^{30}$

- PedsQL Pediatric Pain Questionnaire (5-17 years).

- Functional Status Scale (1 month-17 years). ${ }^{31-33}$

- Paediatric Cerebral Performance Category and the Paediatric Overall Performance Category (1 month-17 years). ${ }^{34-37}$

- Strengths and Difficulties Questionnaire (SDQ; 2-17 years). ${ }^{3839}$

- Child Revised Impact of Events Scale (7-17 years) ${ }^{40-42}$

- Children's Hope Scale (CHS; 8-17 years). ${ }^{43}$

Parent-related measures:

- PedsQL Family Impact Module Version 2.0. ${ }^{44}$

- State-Trait Anxiety Inventory $6 .{ }^{45}$

- Patient Health Questionnaire-4. ${ }^{46}$

- The Post-Traumatic Stress Disorder Checklist (PCL-5) for Diagnostic and Statistical Manual of Mental Disorders-5 (DSM-V). ${ }^{47-49}$

Sibling-related measures:

- PedsQL 4.0 Generic Core Scales (2-17 years). ${ }^{320-29}$

- CHS (8-17 years). ${ }^{43}$

- Multidimensional Assessment of Caring Activities (YC18; 8-17 years)..$^{5051}$

- Positive and Negative Outcomes of Caring (YC20; $8-17$ years $){ }^{51}$

\section{Qualitative study}

The second work package will be a qualitative study involving semistructured interviews with 24 families, split between 3 and 9 months post-PICU discharge. As advocated in the child health literature, a pragmatic and participant-centred approach (based on choice, participation and flexibility) to collecting qualitative data will be employed. Interviews will be conducted with children, parents/legal guardians and siblings either collectively or separately. Interviews will take place at the participants' preferred time and method (eg, face to face, telephone). The use of multiple sources of data will provide contextualised, converging and emerging lines of inquiry.

\section{Sample and recruitment \\ Setting}

Participants will be recruited from at least five PICUs across England chosen to include variation in unit size, case mix, geographical location and patient demographic.

\section{Eligibility criteria}

Participants for this study include: (1) PICU child survivors, (2) parents/legal guardians and (3) siblings.

1. PICU child survivor:(a) Aged 1 month (and $\geq 44$ weeks corrected gestational age) to 17 years at the point of PICU admission; (b) will be discharged from the PICU in next 48 hours; (c) PICU total LOS $\geq 72$ hours at point of discharge in which the patient received PICU therapies for organ dysfunction; (d) at least one parent/ legal guardian ( $\geq 18$ years of age or considered emancipated) living with the potential subject.

2. Parent:(a) Parent or legal guardian; (b) cohabits with the child.

3. Siblings: (a) Aged $\geq 8$ years (at baseline); (b) is a sibling of the children PICU survivor; (c) cohabits with the child PICU survivor for at least $50 \%$ of the time; (d) can independently self-report.

\section{Sample}

Sample size

Quantitative study: we anticipate enrolling 300 children (and their families) from 5 PICUs in equal proportions (60 per centre) over a 6 -month period. Based on previous PICU studies, ${ }^{52}{ }^{53}$ we conservatively estimate a $20 \%$ attrition rate over 1 year. Thus, we anticipate having 1-year outcomes for 240 patients at the end of the study. With 240 participants, we will have high power to detect small/ moderate correlations between early PedsQL measurements (to assess the trajectory of recovery) and other baseline and PICU factors with 1-year PedsQL summary scores. Using a two-sided 0.05 level test, we have $80 \%$ power to detect correlations of 0.18 or larger in magnitude. With 240 participants, we will also have high power to detect moderate differences when comparing two groups using a t-test (eg, comparison of PedsQL summary scores by gender or diagnosis category). In addition, many of the analyses will involve multiple linear regression modelling to adjust for baseline factors or confounding variables. With 240 participants, there is high power for the assessment of modest covariate effects with linear regression. Thus, we anticipate having high power for assessing correlations or linear regression effects as well as for comparing groups with our expected 1-year sample size.

Qualitative study: a stratified sample of up to 24 families (which may include the child, parent and sibling, with 

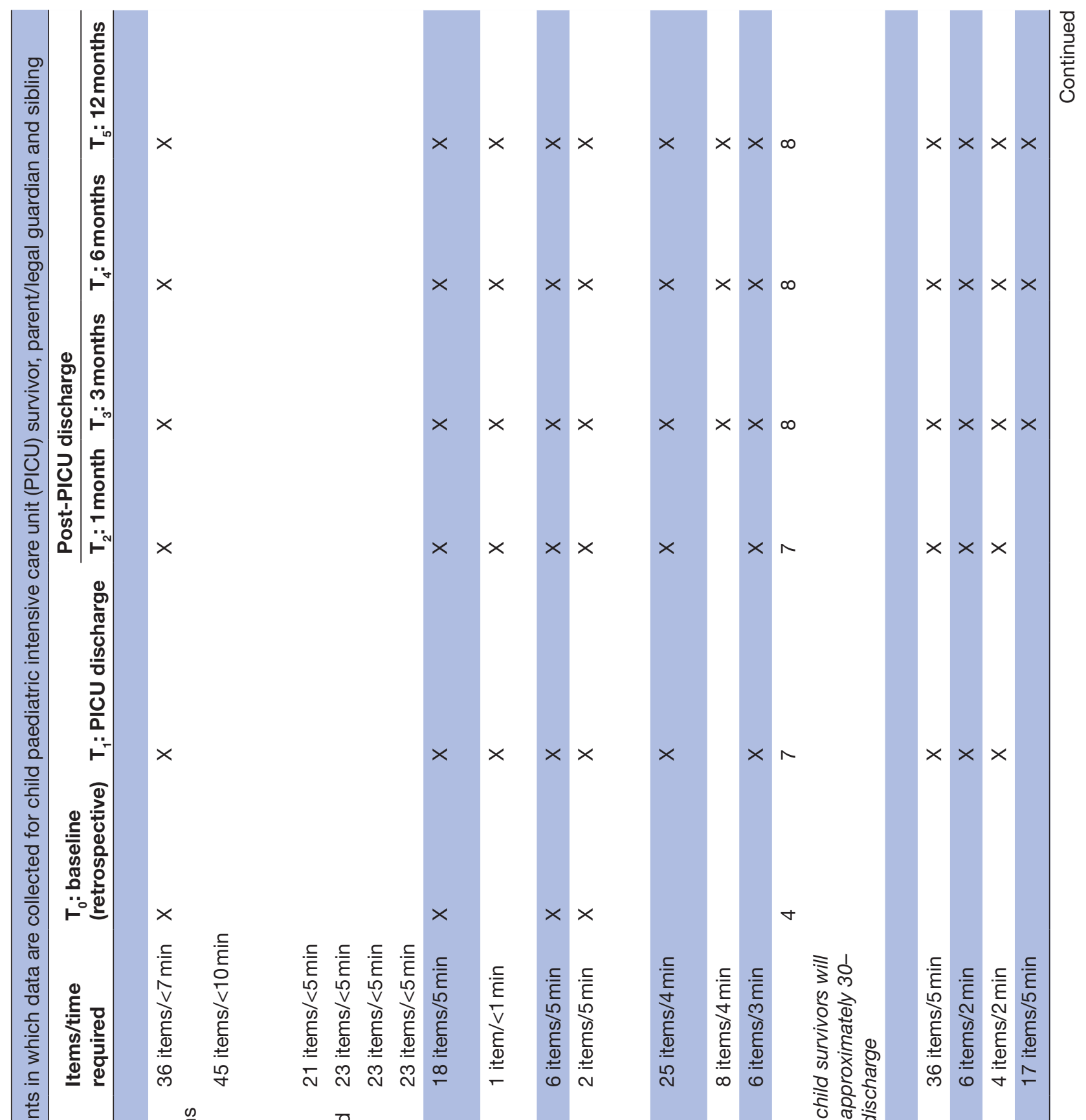

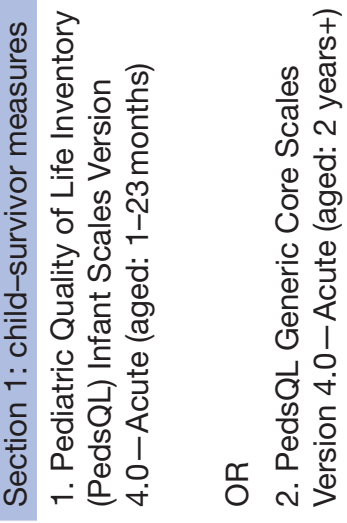

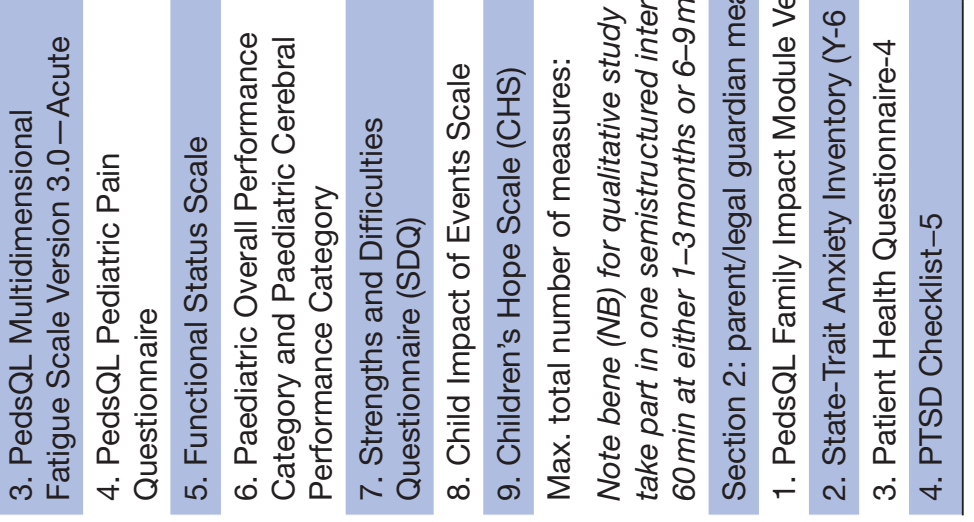

을. 


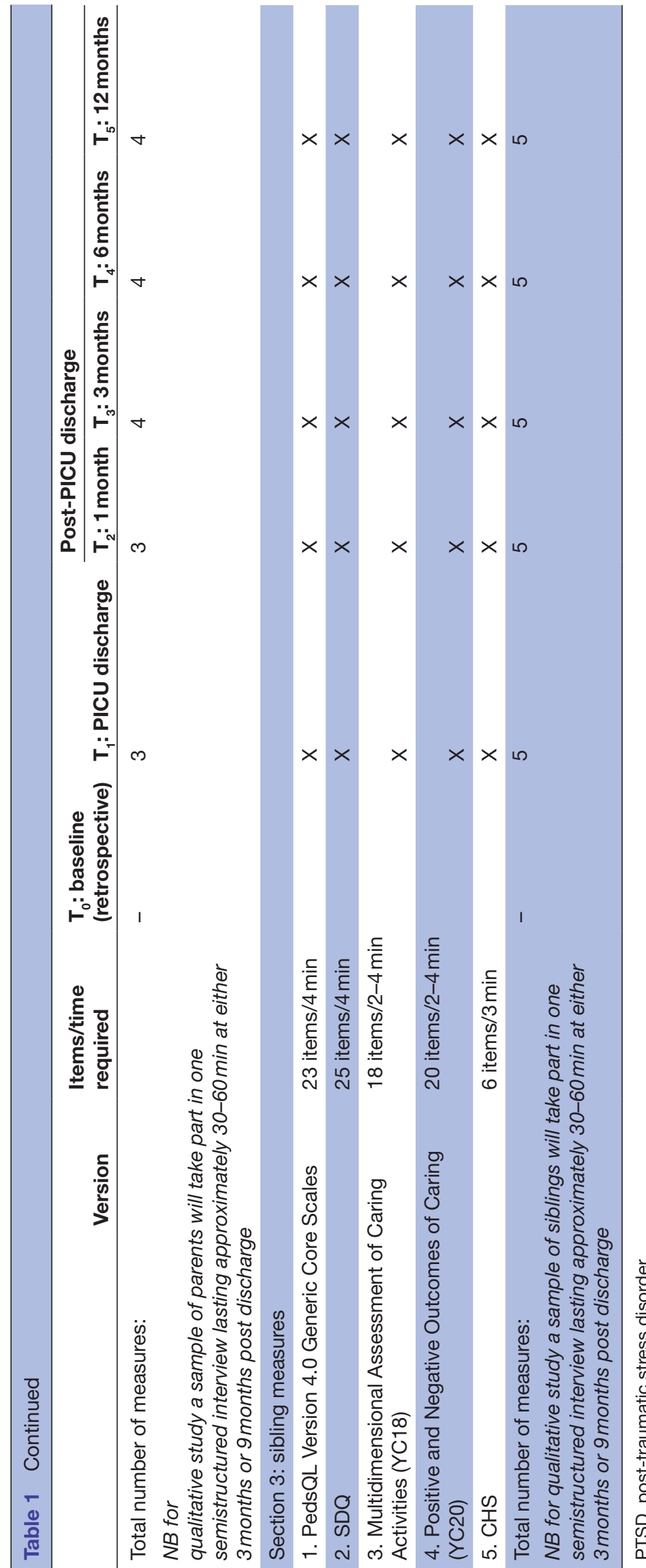

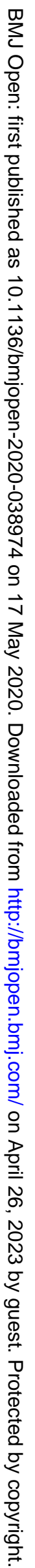


Table 2 Data collection measures, versions and report format according to age and study participant (child paediatric intensive care unit (PICU) survivor, parent/legal guardian or sibling)

\begin{tabular}{lrrrrrrr}
\hline Measure/version (reported by) & $\begin{array}{c}1-12 \\
\text { months }\end{array}$ & $\begin{array}{c}13-23 \\
\text { months }\end{array}$ & $\begin{aligned} 2-4 \\
\text { years }\end{aligned}$ & $\begin{array}{c}5-7 \\
\text { years }\end{array}$ & $\begin{array}{c}8-10 \\
\text { years }\end{array}$ & $\begin{array}{c}11-12 \\
\text { years }\end{array}$ & $\begin{array}{c}13-17 \\
\text { years }\end{array}$ \\
\hline
\end{tabular}

Section 1: child PICU survivor

1. Pediatric Quality of Life Inventory (PedsQL) Infant Scales Version 4.0-Acute

- Infants 1-12 months (Parent reported)

- Infants 13-24 (Parent reported) months

2. PedsQL Generic Core Scales Version 4.0-Acute

- Toddlers

(Parent reported)

- Young child

(Child or parent reported)

- Child

(Child or parent reported)

- Teen

(Child or parent reported)

3. PedsQL Multidimensional Fatigue Scale Version 3.0-Acute

- Toddlers

- Young child

- Child

- Teen
(Parent reported)

(Child or parent reported)

(Child or parent reported)

(Child or parent reported)

4. PedsQL Pediatric Pain Questionnaire

- Young child

(Child or parent reported)

- Child

- Teen

(Child or parent Reported)

(Child or parent reported)

5. Functional Status Scale (Parent reported)

6. Paediatric Cerebral (Parent reported) Performance Category and Paediatric Overall Performance Category

7. Strengths and Difficulties Questionnaire (SDQ)

- 2-4 years old

(Parent reported)

- 4-17years old

(Parent reported)

- 11-17 years old

(Child reported)

8. Child Impact of Events

(Child reported) Scale

9. Children's Hope Scale (CHS)

(Child reported)

Section 2: parent/legal guardian

1. PedsQL Family Impact Module Version 2.0-Acute

2. State-Trait Anxiety Inventory (Y-6 item)

3. Patient Health Questionnaire-4

4. PTSD Checklist (PCL) -5

Section 3: sibling

1. PedsQL Generic Core Scales Version 4.0-Acute

- Child
PICU survivor participant age

$x$
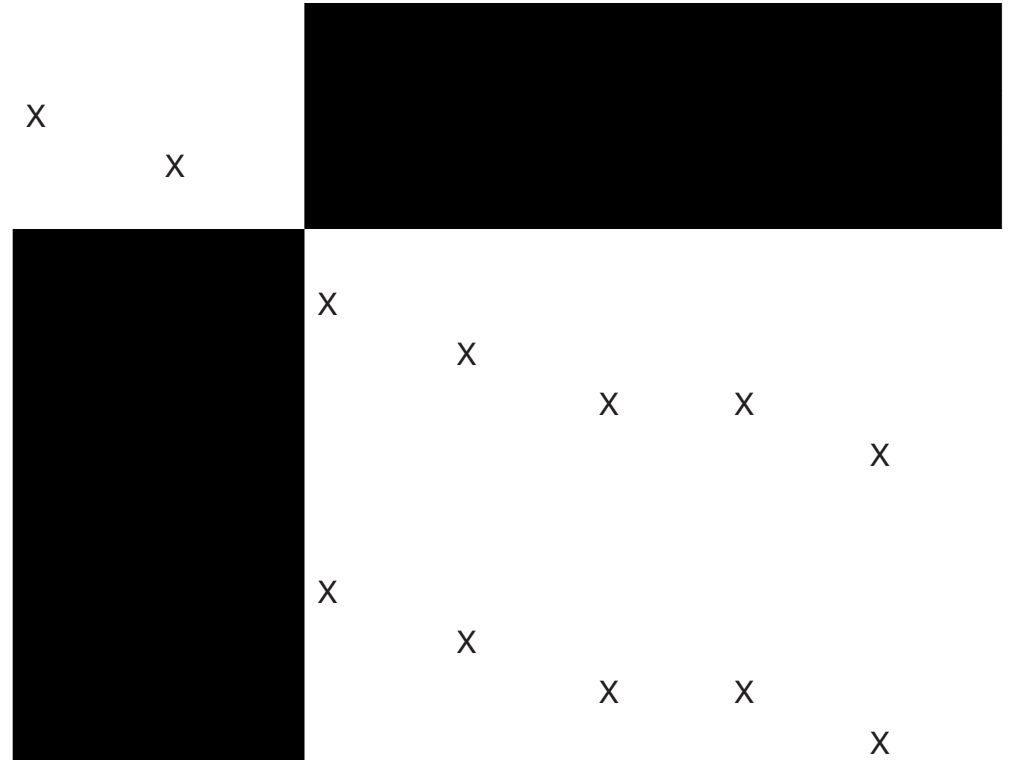

$x$

$\mathrm{X}$

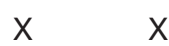

$X$

$x$

$x \quad x$

$\mathrm{x}$

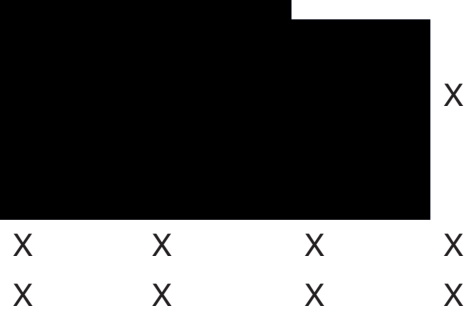

x $\quad x$

$\begin{array}{lll}x & x & x\end{array}$

$x \quad x \quad x$

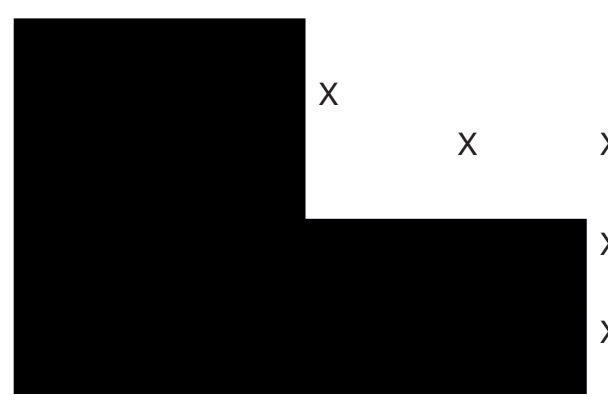

$x \quad x$

$x \quad x$

Parent/legal guardian

(Parent reported) $\mathrm{X}$

(Parent reported) $\quad X$

(Parent reported) $\quad X$

(Parent reported) $\quad X$

Sibling participant age

(Child reported) X $\quad$ X 
Table 2 Continued

\begin{tabular}{|c|c|c|c|c|c|c|}
\hline Measure/version (reported by) & $\begin{array}{r}13-23 \\
\text { months }\end{array}$ & $\begin{array}{r}2-4 \\
\text { years }\end{array}$ & $\begin{array}{r}5-7 \\
\text { years }\end{array}$ & $\begin{array}{l}8-10 \\
\text { years }\end{array}$ & $\begin{array}{l}11-12 \\
\text { years }\end{array}$ & $\begin{array}{l}13-17 \\
\text { years }\end{array}$ \\
\hline$>$ Teen & & \multicolumn{3}{|c|}{ (Child reported) } & & $x$ \\
\hline \multicolumn{7}{|l|}{ 2. SDQ } \\
\hline - 4-17years old & & (Paren & eported) & $x$ & & \\
\hline - 11-17years old & & (Child & ported) & & $x$ & $x$ \\
\hline 3. Multidimensional Assessment of Caring Activities (YC18) & & (Child & ported) & $\mathrm{x}$ & $\mathrm{x}$ & $\mathrm{X}$ \\
\hline 4. Positive and Negative Outcomes of Caring (YC20) & & (Child & ported) & $\mathrm{x}$ & $\mathrm{x}$ & $\mathrm{x}$ \\
\hline 5. $\mathrm{CHS}$ & & (Child & ported) & $x$ & $x$ & $x$ \\
\hline
\end{tabular}

PTSD, post-traumatic stress disorder.

a maximum of 72 participants in total) will be enrolled into the qualitative interviews. This sample size will capture diverse perspectives around support needs and is expected to achieve data saturation in the qualitative analysis. ${ }^{54}$

\section{Sampling technique}

Quantitative study sampling technique: a consecutive sampling strategy will be employed.$^{55}$ Each site will screen daily over a 12-month period and invite all eligible children to participate in the study. Data from screening logs, including refusal to participate and admission numbers at each site, will be collected and used to contextualise the reporting of the analysis. In order to recruit a sample that is representative of the PICU populous, a sampling frame based on age and diagnosis reported from PICANet data $^{1}$ will be used. This frame will be used to guide the recruitment of participants recruited into the study and is outlined in table 3 .

Qualitative study sampling technique: two cohorts of 12 families (including the child, parent and a sibling) will be selected using a stratified sampling approach based on the child's PedsQL score at 1-month post-PICU discharge and 6-month post-PICU discharge. Stratification using previously reported norms for PedsQL as well as variation in relation to geographical locality, PICU presenting condition, age and ethnicity will be sought.

\section{Study procedures}

Quantitative study

Over a 6-month period, each site will screen daily the children admitted to PICU and invite all eligible children to participate in the study. Site investigators (or their designated nominee) who are part of the PICU clinical care team will determine eligibility.

In line with feedback from PPI work in the development of this study, each participant (aged $\geq 5$ years) will be provided with a single $£ 15$ gift voucher as a token of appreciation for participating in the study. Vouchers will be provided to all participants on the completion of the study data collection period ( $\mathrm{T} 6-12$ month's post-PICU discharge).

\section{Qualitative study}

For the qualitative study, participants will be identified from PedsQL scores of the child participant at 1-month post-PICU discharge and 6-month post-PICU discharge. The identification and recruitment process are summarised in figure 3 and will follow a systematic process:

1. Child participant PedsQL scores will be collected and submitted by sites onto REDCap Cloud.

2. The chief investigator will review the scores and stratify the sample based whether the PedsQL score is within

Table 3 Proposed sampling frame for paediatric intensive care unit survivor participant recruitment

\section{Diagnosis}

\begin{tabular}{llllll}
\hline Age (years) & Cardiovascular (28.1\%) & Neurological (10.7\%) & Respiratory (29.2\%) & $\begin{array}{l}\text { Other* } \\
\text { (32\%) }\end{array}$ & Total \\
\hline $0(55 \%)$ & 47 & 19 & 48 & 53 & 167 \\
\hline $1-5(25.2 \%)$ & 21 & 8 & 23 & 25 & 77 \\
\hline $6-10(9.7 \%)$ & 8 & 3 & 8 & 9 & 28 \\
$\geq 11(10.3 \%)$ & 8 & 3 & 8 & 10 & 28 \\
Total & 84 & 33 & 87 & 63 & 300 \\
\hline
\end{tabular}

*Including: blood/lymphatic; body wall and cavities; endocrine/metabolic; trauma; oncology; musculoskeletal; multisystem; infection; gastrointestinal. 


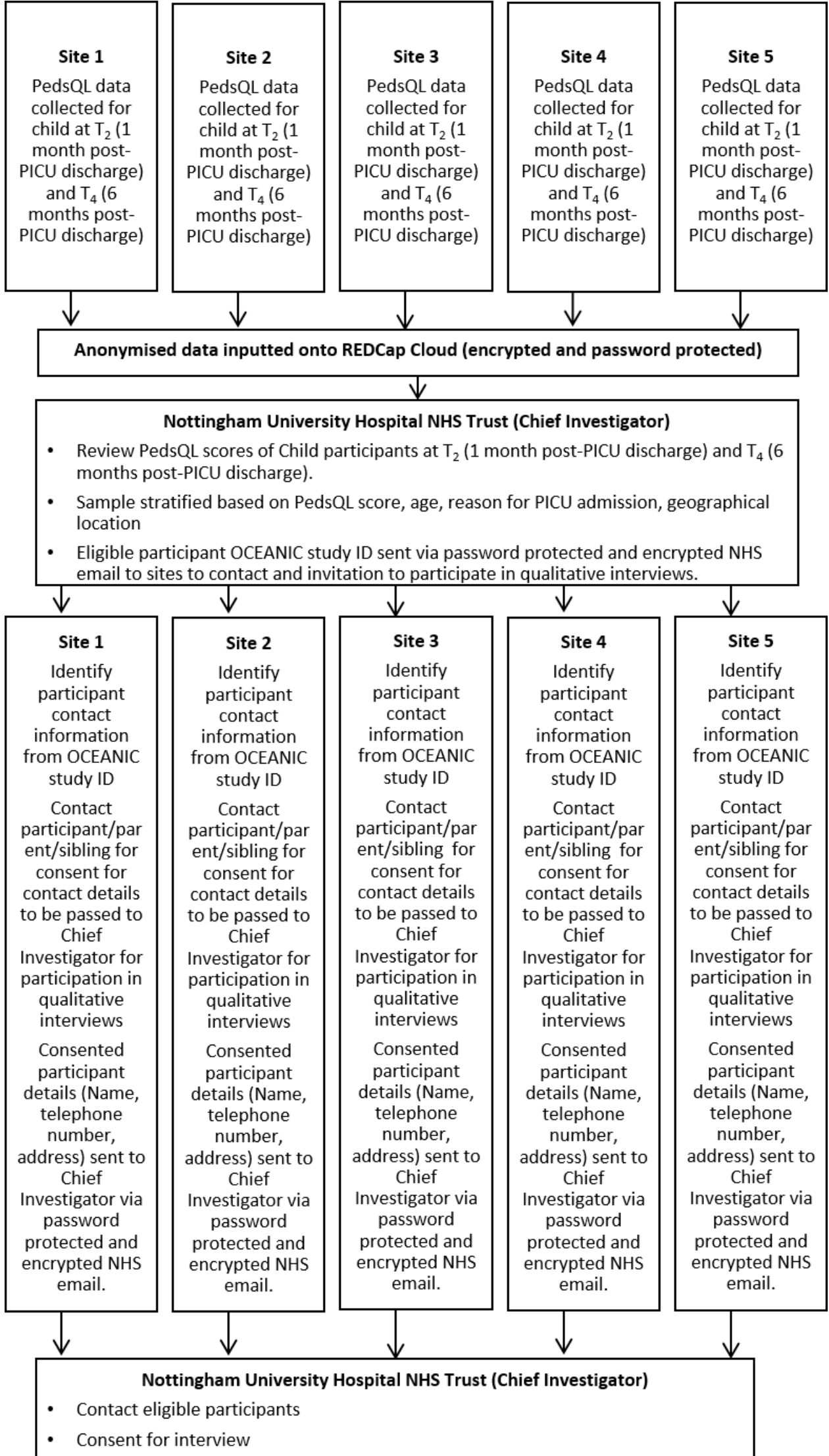

Figure 3 Identification (ID) and recruitment of participants for Qualitative Study. NHS, National Health Service; OCEANIC, Outcomes of ChildrEn and fAmilies in the first year after paediatric Intensive Care; PedsQL, Pediatric Quality of Life Inventory; PICU, paediatric intensive care unit. 
1,2 or $>2$ SD from the published norms, selecting at least 4 children for each group at 1-month post-PICU discharge and 6-month post-PICU discharge. To maximise diversity in families (child, parent and sibling) interviewed, where possible participants will be selected based on geographical locality, PICU presenting condition, age and ethnicity.

3. The study ID of potential participants will be sent to sites, who will then contact the family directly, requesting consent to receive contact from the chief investigator/study researcher.

4. The chief investigator/study researcher will contact families that have agreed to being contacted, to consent for qualitative interviews and to arrange suitable date, time and location.

\section{Analyses}

Quantitative study data analysis

Descriptive statistics will be presented for demographic information, and medical history. All child, parent and sibling-related measures will be calculated, including means, SD, medians and IQRs for continuous variables and frequency counts and percentages for categorical variables. Data will be examined for normality, outliers and systematic missing data. Transformations will be undertaken as needed.

Analyses related to specific objectives include the following:

Objective 1: To describe the physical, cognitive, emotional and social health outcomes and trajectory of recovery in children postPICU discharge. The primary aim is to explore child PICU survivors' health outcomes and trajectory of recovery over the first-year post-PICU discharge. PICU survivors' health outcomes will be compared with published population means from the general and chronically ill populations using t-tests or Mann-Whitney test as appropriate. For the longitudinal data, correlations will be assessed between time points using Spearman correlations and a linear mixed regression model with random subject effects will be used to analyse trajectories over time. In case of lack of normality, the non-parametric longitudinal approach will be implemented.

Objective 2: To determine the baseline and PICU factors associated with impaired outcomes. To identify factors associated with impaired health outcomes among PICU survivors, correlation analyses followed by principle component analysis (PCA) will be applied to identify covariates for the regression modelling. For categorised recovery over 1-year post-PICU discharge, mixed effect logistic regression will be applied. Variables will be entered using backward stepwise approach to control for collinearity. Model performance will be assessed using sensitivity, specificity, positive predicted value, negative predicted value and Area Under Curve Reciever Operating Characteristics (AUCROC) values. Bootstrapping through K-fold approach will be applied to ensure better modelling.

Objective 3: To explore the longitudinal emotional and social health outcomes of parents and siblings. Parent and sibling emotional- and social health outcomes will be compared with published means using t-tests or Mann-Whitney test as appropriate. PICU survivor and sibling PedsQL summary scores and SDQ scores will also be compared using paired t-tests or Wilcoxon Signed Rank test.

Graphical analyses will be performed to display the trajectories of health outcomes over time in our populations of critically ill children. Multiple linear and logistic regression methods will be used to explore the effects of primary diagnosis (eg, respiratory, cardiovascular), PICU LOS category, and site, to predict outcomes. We will explore whether adjustment for sex, race/ethnicity or site affects study inferences through the use of mixed effects and generalised estimating equations models. Finally, we will also explore the use of classification and regression trees with recursive partitioning, PCA, factor analysis and machine learning methods to help describe subgroups of patients with similar trajectories of outcome.

\section{Qualitative study data analysis}

Audio recorded interview data will be transcribed verbatim with all participant identifiable information removed. Transcription will be conducted by a service approved by Nottingham University Hospitals National Health Service (NHS) Trust Research and Innovation Department. Confidentiality agreements will be completed. Transcripts will be imported into NVivo V.12, for sorting, coding and categorising of the data.

Qualitative data will be analysed using the adapted five-stage framework analysis process to achieve objective 4 ; identification of the care and support needs of children, their parents and siblings. The five stages of framework analysis comprise (1) familiarisation with the data through reading full transcripts; (2) development of a theoretical framework through identification of recurring and important themes; (3) indexing and pilot charting; (4) summarising data in an analytical framework; and (5) synthesising data by mapping and interpreting. ${ }^{56}$ Stages 1-4 will be conducted separately for respondent type (children, parents or siblings) to enable specific care and support needs to be identified and summarised. Stage 5 will then allow for data to be compared and contrasted across the respondent groups (child, parent, sibling), child's PedsQL score $(<1,2$, or $>2$ SD from published norms), and time points (1-3 months or 6-9 months post-PICU discharge).

\section{Patient and Public Involvement}

Underpinned by the best principles of National Institute for Health Research (NIHR) INVOLVE (https://www. invo.org.uk/), children, young people (CYP) and families have been integral to the development of this study. In 2017, the chief investigator (Dr Manning) and co-investigator (Professor Latour) organised the UK's first symposium on aftercare and rehabilitation following PICU and engaged with over 60 PICU clinicians, an ex-PICU patient and family members. Feedback identified that: a prospective longitudinal cohort study to further understand 
the outcomes for CYP and their families post-PICU was needed; and the collection of data at multiple time points over the first year would have value for CYP and their families, health professionals and research to direct the development of future interventions.

Further PPI has been undertaken with 11 parents (seven mothers and four fathers), 4 siblings (aged 9-13 years) and 3 CYP PICU survivors (aged 11-17 years) from the East and West Midlands. Participants' varied in ethnicity and family composition, and reasons for admissions to different PICUs. The proposed study was regarded as addressing an important topic. Respondents main concerns included: the potential to trigger negative reactions from participation; the collection of information pertaining to the preintensive care unit state and the difficulty of considering their own emotional well-being when their focus is on their child's survival. Suggestions to address these included: certificates and vouchers to thank participants, flexibility in the method of data collection, linking up with existing support services to build reminders and removing reference to scores within the survey/s. Making the purpose of the research more visible through study website and social media would help parents' make decisions about participating and keeping updated with the study.

As part of this study, we will continue to have meaningful advice and input from PPI. An advisory group has been assembled consisting of a young person that has been critically ill, parents and carers of children that have experienced critical illness/injury, and a sibling of a critical illness survivor. It is proposed that this group will have at least 6 monthly meetings to ensure they have continued and active involvement in: the management of the research; developing participant information resources; contributing to the study report and dissemination of research findings.

\section{ETHICS AND DISSEMINATION \\ Ethics}

This research includes recruitment of seriously ill children on a PICU and a parent and sibling. It concerns a challenging topic requiring great skill and sensitivity in data collection. The study is being carried out by an experienced research team with clinical and research expertise in children and young people who are seriously ill. Research staff will have also received one-to-one protocol training with the CI. We will ensure the first approach is from a member of the child's usual care team, and is sensitive to the situation and status of the child.

PPI is central to this project and in ensuring that it remains grounded in the experiences of patients. The associated participant facing materials will be carefully developed (with age specific information sheets and consent/assent forms) and these will be reviewed by a PPI panel. The information sheets clearly state that discussing the experience of serious illness may be distressing, and we will ask participants to consider carefully how they feel about this prospect before deciding to take part.

\section{Consent/assent}

Eligible participants will be given at least 24 hours to consider whether they wish to participate in the study. It will be made clear to the parents that they will be free to withdraw their consent for their own and/or their child's participation in the study at any time without this having any impact on their child's care. The majority of children will be sedated and on a ventilator at recruitment, therefore will be unable to provide informed consent/assent.

For those children unable to provide consent/assent at the time of enrolment into the study, consent will be obtained from their parent/legal guardian. Efforts will be made to then consent/assent the child once they are able to (eg, have the cognitive capacity) by the site teams. In the unlikely event that a child does not wish to participate (and the parent has consented for the child), the child's wishes will be upheld and the parent/sibling will be withdrawn from the study.

\section{Interviews}

We recognise that the discussing/recollecting a potentially difficult experience (the PICU admission) and any ongoing health and care needs may be upsetting for survivors/parents/families. ${ }^{10}$ Therefore, all interviews will be conducted by the chief investigator or the OCEANIC Research Fellow, who both have previous experience of conducting interviews with children and families on sensitive issues. Interviews will be semistructured over 30-60 min with appropriate breaks if necessary. Interviews will allow participants to explore any issues in depth, which in itself may provide opportunity for issues, feelings and emotions to be discussed. This will be facilitated by creative/child centred data collection techniques that are sensitive to exploring potentially emotive events, in a constructive manner. Families will be given the choice whether they would like to have the interview separately (child, parent and sibling) or collectively.

It will be made clear to participants at the outset that the interview can be stopped at any time should they wish. Furthermore, if the child participant, their parent/legal guardian, or sibling becomes visibly upset during the interview, the investigator will:

1. Invite the parent/legal guardian (if present) to console the child/sibling, (if not already doing so).

2. Offer to temporarily stop or terminate the visit.

3. Respect the decision made by the participant to stop/ carry on the interview.

All visits with children ( $<16$ year olds) will be conducted with the parent/legal guardian present. In cases where it is not possible for parents to be present or the child specifically requests for them not to be present, a second investigator from the study team will be present. All the study investigators have an enhanced Disclosure and Barring Service check. All investigators conducting the qualitative interviews are registered with Nursing and Midwifery 
Council (UK, first level) and are therefore bound by codes of professional conduct and have a professional obligation to share information with other agencies (ie, social services), if an interview participant discloses information that relates to safeguarding or child protection.

\section{Ethical review}

The West Midlands-The Black Country NHS Research Ethics Committee has reviewed the study protocol and provided favourable opinion (Ref: 19/WM/0290). The Health Research Authority has also approved the protocol (IRAS: 269642). This study has been externally peer reviewed and awarded funding through a competitive process through the National Institute for Health Research (NIHR) (ICA-CL-2018-04-ST2-009). The study has been registered in International Standard Randomised Controlled Trials Number 28072812.

\section{Dissemination}

Despite advances to the evidence base, a comprehensive understanding of PICU morbidity among survivors after PICU discharge remains limited. Historically, studies have focused on specific populations and/or diseases (such as prematurity, congenital heart disease, long-stay patients) rather than on issues experienced by the post-PICU discharge population as a whole ${ }^{32}{ }^{57-63}$ Moreover, these studies to date have examined variable outcomes (such as functional status, health-related qualify of life, psychological well-being, adaptive behaviours) at a single time point, ${ }^{32} 57-63$ with few studies considering the patient's pre-PICU status. Collectively, this heterogeneity in scope severely limits understanding of morbidities experienced by children who survive critical illness, and their trajectories. ${ }^{27}$

While there is a definite need to understand the longterm outcome trajectories of children and families, the scope and purpose of this research are to address this critical gap by being the first study to provide a comprehensive and contemporary understanding of the outcomes of children and families in the first-year post-PICU admission. This will allow for health deficits across a spectrum of domains to be identified. It will provide a better understanding of those at risk of morbidity post-PICU admission, when this manifests, its natural history and any factors that could be modified to improve outcomes. Novel and contemporary insights into the outcomes of children and their family will be established through the study findings, which has been recognised as global priority area for PICU research. Moreover, this study will enhance understanding of the health outcomes of underresearched groups within the PICU populous including those very young children ( $<2$ years), as well as those with communication/developmental impairments. Collectively, characterisation of the longitudinal recovery of children, their parents and siblings post-PICU discharge will allow interventions to be identified to prevent or mitigate morbidity and therefore have the potential to optimise the outcomes and lives of children and their families.
Findings will impact on the delivery and configuration of current services, as well as having the potential to inform the development of new models of care that improve the quality of services for patients and families.

The dissemination strategy will be multifaceted to ensure findings are reported in a timely and relevant manner to key stakeholders that include patients and the public, healthcare professionals, commissioners and policy-makers, and academics. Findings will be reported within a funder report (accessible through the NIHR Academy website), professional journals and in highquality peer-reviewed, open-access journals. In addition, members of the PPI advisory group will assist in composing a summary which will be distributed to national parent support groups and charities. Key findings will also be posted on institutional websites and social media.

\section{Author affiliations}

${ }^{1}$ Children and Young People Health Research, School of Health Sciences, University of Nottingham, Nottingham, UK

${ }^{2}$ Nottingham Children's Hospital, Nottingham University Hospitals NHS Trust, Nottingham, Nottinghamshire, UK

${ }^{3}$ Health Data Research UK, University of Nottingham, Nottingham, UK ${ }^{4}$ School of Nursing and Midwifery, University of Plymouth, Plymouth, UK ${ }^{5}$ Nursing Department, Hunan Children's Hospital, Changsha, Hunan, China ${ }^{6}$ Department of Family and Community Health, School of Nursing, University of Pennsylvania, Philadelphia, Pennsylvania, USA

${ }^{7}$ Anesthesia and Critical Care Medicine, Perelman School of Medicine, University of Pennsylvania, Philadelphia, Pennsylvania, USA

${ }^{8}$ The Research Institute, Children's Hospital of Philadelphia, Philadelphia, PA, USA

${ }^{9}$ Department of Health Sciences, University of Leicester, Leicester, UK

${ }^{10}$ Advanced Data Analysis Centre, University of Nottingham, Nottingham, UK

${ }^{11}$ Division of Pediatric Critical Care Medicine, Department of Pediatrics, University of Washington, Seattle, Washington, USA

${ }^{12}$ Centre for Child Health, Behaviour, and Development, Seattle Children's Research Institute, Seattle, Washington, USA

${ }^{13}$ Ingram School of Nursing, McGill University Faculty of Medicine, Montreal, Quebec, Canada

${ }^{14}$ Centre for Outcomes Research \& Evaluation, Research Institute of the McGill University Health Centre, Montreal, Quebec, Canada

${ }^{15}$ Paediatric Psychology Service, St Georges University Hospitals NHS Foundation Trust, London, UK

${ }^{16}$ Population Health Research Institute, University of London St George's, London, UK ${ }^{17}$ Section of Pediatric Critical Care, Department of Pediatrics, University of Chicago, Chicago, Illinois, USA

${ }^{18}$ School of Health Sciences, University of Nottingham, Nottingham, Nottinghamshire, UK

Twitter Joseph C Manning @josephcmanning

Acknowledgements The OCEANIC study was adapted from the PICS-p Study protocol developed by MC, RSW, GC, JR, NP and JCM. The authors would like to acknowledge Julie Menzies (Nurse Researcher, Birmingham Children's Hospital) in supporting the Patient and Public Involvement activities undertaken as part of the development of the OCEANIC study protocol. Furthermore, they would like to acknowledge Francois Arvin-Berod (Data Manager, Research and Innovation, Nottingham University Hospitals NHS Trust) who supported the development of the OCEANIC data capture and management through the REDCap Cloud.

Contributors JCM is the chief investigator for the OCEANIC study. JCM, JL, MC, ESD, TJ, PRQ, RSW, JR, GC, NP and JC made a substantial contribution to the conceptualisation and design of the study. JCM, AL and EP drafted the first version of the manuscript. All authors critically revised the manuscript for important intellectual content, gave approval of the final version to be published, and agreed to be accountable for all aspects of the work.

Funding JCM is recipient of a National Institute for Health Research ICA Clinical Lectureship (ICA-CL-2018-04-ST2-009) which is funding this research study. 
Competing interests None declared.

Patient and public involvement Patients and/or the public were involved in the design, or conduct, or reporting, or dissemination plans of this research. Refer to the Methods section for further details.

Patient consent for publication Not required.

Ethics approval National Health Services Research Ethics Committee [Ref: 19/ WM/0290]

Provenance and peer review Not commissioned; peer reviewed for ethical and funding approval prior to submission.

Open access This is an open access article distributed in accordance with the Creative Commons Attribution 4.0 Unported (CC BY 4.0) license, which permits others to copy, redistribute, remix, transform and build upon this work for any purpose, provided the original work is properly cited, a link to the licence is given, and indication of whether changes were made. See: https://creativecommons.org/ licenses/by/4.0/.

ORCID iD

Joseph C Manning http://orcid.org/0000-0002-6077-4169

\section{REFERENCES}

1 PICANet. Annual report: summary report. Leeds: Universities of Leeds and Leicester, 2017.

2 Namachivayam P, Shann F, Shekerdemian L, et al. Three decades of pediatric intensive care: who was admitted, what happened in intensive care, and what happened afterward. Pediatr Crit Care Med 2010;11:549-55.

3 Pollack MM, Holubkov R, Funai T, et al. Pediatric intensive care outcomes: development of new morbidities during pediatric critical care. Pediatr Crit Care Med 2014;15:821-7.

4 Shudy M, de Almeida ML, Ly S, et al. Impact of pediatric critical illness and injury on families: a systematic literature review. Pediatrics 2006;118 Suppl 3:S203-18.

5 Abela KM, Wardell D, Rozmus C, et al. Impact of pediatric critical illness and injury on families: an updated systematic review. J Pediatr Nurs 2020;51:21-31.

6 Rennick JE, Rashotte J. Psychological outcomes in children following pediatric intensive care unit hospitalization: a systematic review of the research. J Child Health Care 2009;13:128-49.

7 Davydow DS, Richardson LP, Zatzick DF, et al. Psychiatric morbidity in pediatric critical illness survivors: a comprehensive review of the literature. Arch Pediatr Adolesc Med 2010;164:377-85.

8 Manning JC, Hemingway P, Redsell SA. Long-Term psychosocial impact reported by childhood critical illness survivors: a systematic review. Nurs Crit Care 2014;19:145-56.

9 Nelson LP, Gold Jl. Posttraumatic stress disorder in children and their parents following admission to the pediatric intensive care unit: a review. Pediatr Crit Care Med 2012;13:338-47.

10 Manning JC, Hemingway P, Redsell SA. Stories of survival: children's narratives of psychosocial well-being following paediatric critical illness or injury. J Child Health Care 2017;21:236-52.

11 Needham DM, Davidson J, Cohen $\mathrm{H}$, et al. Improving long-term outcomes after discharge from intensive care unit: report from a stakeholders' conference. Crit Care Med 2012;40:502-9.

12 Spragg RG, Bernard GR, Checkley W, et al. Beyond mortality: future clinical research in acute lung injury. Am J Respir Crit Care Med 2010;181:1121-7.

13 Turnbull AE, Rabiee A, Davis WE, et al. Outcome measurement in ICU survivorship research from 1970 to 2013: a scoping review of 425 publications. Crit Care Med 2016;44:1267.

14 Reay H, Arulkumaran N, Brett SJ. Priorities for future intensive care research in the UK: results of a James Lind alliance priority setting partnership. J Intensive Care Soc 2014;15:288-96.

15 National Institute for Health and Care Excellence. Rehabilitation after critical illness in adults. Quality Standard [QS158]. London NICE, 2017.

16 Manning JC, Hemingway P, Redsell SA. Survived so what? identifying priorities for research with children and families postpaediatric intensive care unit. Nurs Crit Care 2018;23:68-74.

17 Manning JC, Scholefield BR, Popejoy E, et al. Paediatric intensive care follow-up provision in the United Kingdom and Republic of Ireland. Nurs Crit Care 2020. doi:10.1111/nicc.12510. [Epub ahead of print: 23 Apr 2020].

18 Watson RS, Choong K, Colville G, et al. Life after critical illness in Children-Toward an understanding of pediatric Post-intensive care syndrome. J Pediatr 2018;198:16-24.
19 Manning JC, Pinto NP, Rennick JE, et al. Conceptualizing post intensive care syndrome in Children-The PICS-p framework. Pediatr Crit Care Med 2018;19:298-300.

20 Seid M, Limbers CA, Driscoll KA, et al. Reliability, validity, and responsiveness of the pediatric quality of life inventory (PedsQL) generic core scales and asthma symptoms scale in vulnerable children with asthma. J Asthma 2010;47:170-7.

21 Seid M, Varni JW, Gidwani P, et al. Problem-Solving skills training for vulnerable families of children with persistent asthma: report of a randomized trial on health-related quality of life outcomes. $J$ Pediatr Psychol 2010;35:1133-43.

22 Varni JW, Seid M, Knight TS, et al. The PedsQL 4.0 generic core scales: sensitivity, responsiveness, and impact on clinical decisionmaking. J Behav Med 2002;25:175-93.

23 Varni JW, Seid M, Smith Knight T, et al. The PedsQL in pediatric rheumatology: reliability, validity, and responsiveness of the pediatric quality of life inventory generic core scales and rheumatology module. Arthritis Rheum 2002;46:714-25.

24 Mistry RD, Stevens MW, Gorelick MH. Health-Related quality of life for pediatric emergency department febrile illnesses: an evaluation of the pediatric quality of life inventory 4.0 generic core scales. Health Qual Life Outcomes 2009;7:5.

25 Brandow AM, Brousseau DC, Pajewski NM, et al. Vaso-occlusive painful events in sickle cell disease: impact on child well-being. Pediatr Blood Cancer 2010;54:92-7.

26 Aspesberro F, Fesinmeyer MD, Zhou C, et al. Construct validity and responsiveness of the pediatric quality of life inventory 4.0 generic core scales and infant scales in the PICU. Pediatr Crit Care Med 2016;17:e272-9.

27 Ong C, Lee JH, Leow MKS, et al. Functional outcomes and physical impairments in pediatric critical care survivors: a scoping review. Pediatr Crit Care Med 2016;17:e247-59.

28 Janssens L, Gorter JW, Ketelaar M, et al. Health-Related quality-oflife measures for long-term follow-up in children after major trauma. Qual Life Res 2008;17:701-13.

29 Eiser C, Morse R. The measurement of quality of life in children: past and future perspectives. J Dev Behav Pediatr 2001;22:248-56.

30 Varni JW, Burwinkle TM, Katz ER, et al. The PedsQL in pediatric cancer: reliability and validity of the pediatric quality of life inventory generic core scales, multidimensional fatigue scale, and cancer module. Cancer 2002:94:2090-106.

31 Aspesberro F, Mangione-Smith R, Zimmerman JJ. Health-Related quality of life following pediatric critical illness. Intensive Care Med 2015;41:1235-46.

32 Pollack MM, Holubkov R, Glass P, et al. Functional status scale: new pediatric outcome measure. Pediatrics 2009;124:e18-28.

33 Pollack MM, Holubkov R, Funai T, et al. Simultaneous prediction of new morbidity, mortality, and survival without new morbidity from pediatric intensive care: a new paradigm for outcomes assessment. Crit Care Med 2015;43:1699-709.

34 Fiser DH, Long N, Roberson PK, et al. Relationship of pediatric overall performance category and pediatric cerebral performance category scores at pediatric intensive care unit discharge with outcome measures collected at hospital discharge and 1- and 6-month follow-up assessments. Crit Care Med 2000;28:2616-20.

35 Fiser DH, Tilford JM, Roberson PK. Relationship of illness severity and length of stay to functional outcomes in the pediatric intensive care unit: a multi-institutional study. Crit Care Med 2000;28:1173-9.

36 Fiser $\mathrm{DH}$. Assessing the outcome of pediatric intensive care. J Pediatr 1992;121:68-74.

37 Fuchs S, Lewis RJ. Tools for the measurement of outcome after minor head injury in children: summary from the ambulatory pediatric Association/EMSC outcomes research conference. Acad Emerg Med 2003;10:368-75.

38 Goodman R. Psychometric properties of the strengths and difficulties questionnaire. J Am Acad Child Adolesc Psychiatry 2001;40:1337-45.

39 Goodman R. The strengths and difficulties questionnaire: a research note. J Child Psychol Psychiatry 1997;38:581-6.

40 Anxiety YW. Depression and post-traumatic stress in childhood. In: Sclare I, ed. Child psychology Portfolio. Windsor, UK: NFER-Nelson, 1997: 35-8.

41 Colville G, Pierce C. Patterns of post-traumatic stress symptoms in families after paediatric intensive care. Intensive Care Med 2012;38:1523-31.

42 Perrin S, Meiser-Stedman R, Smith P. The children's revised impact of event scale (cries): validity as a screening instrument for PTSD. Behav Cogn Psychother 2005;33:487-98.

43 Snyder CR, Hoza B, Pelham WE, et al. The development and validation of the children's hope scale. J Pediatr Psychol 1997;22:399-421. 
44 Varni JW, Sherman SA, Burwinkle TM, et al. The PedsQL family impact module: preliminary reliability and validity. Health Qual Life Outcomes 2004;2:55.

45 Marteau TM, Bekker $\mathrm{H}$. The development of a six-item short-form of the state scale of the Spielberger State-Trait anxiety inventory (STAI). Br J Clin Psychol 1992;31:301-6.

46 Kroenke K, Spitzer RL, Williams JBW, et al. An ultra-brief screening scale for anxiety and depression: the PHQ-4. Psychosomatics 2009;50:613-21.

47 Weathers FW, Litz BT, Keane TM, et al. The PTSD checklist for DSM-5 (PCL-5. US: Department of Veteran Affairs, National Center for PTSD, 2013. https://www.ptsd.va.gov/professional/assessment/ adult-sr/ptsd-checklist.asp

48 Blevins CA, Weathers FW, Davis MT, et al. The posttraumatic stress disorder checklist for DSM-5 (PCL-5): development and initial psychometric evaluation. J Trauma Stress 2015;28:489-98.

49 Bovin MJ, Marx BP, Weathers FW, et al. Psychometric properties of the PTSD checklist for diagnostic and statistical manual of mental Disorders-Fifth edition (PCL-5) in veterans. Psychol Assess 2016;28:1379-91.

50 Joseph S, Becker S, Becker F, et al. Assessment of caring and its effects in young people: development of the multidimensional assessment of caring activities checklist (MACA-YC18) and the positive and negative outcomes of caring questionnaire (PANOCYC20) for young carers. Child Care Health Dev 2009;35:510-20.

51 Joseph S, Becker F, Becker S. Manual for measures of caring activities and outcomes for children and young people. Nottingham: The University of Nottingham, 2009.

52 Khetani MA, Albrecht EC, Jarvis JM, et al. Determinants of change in home participation among critically ill children. Dev Med Child Neurol 2018;60:793-800.
53 Rennick JE, Dougherty G, Chambers C, et al. Children's psychological and behavioral responses following pediatric intensive care unit hospitalization: the caring intensively study. BMC Pediatr 2014;14:276.

54 Morse JM. Determining sample size qualitative health research. . Sage, 2000: 10. 3-5.

55 Daniel J. Sampling essentials: practical guidelines for making sampling choices. Thousand Oaks, California, 2012. http://methods. sagepub.com/book/sampling-essentials

56 Ward DJ, Furber C, Tierney S, et al. Using framework analysis in nursing research: a worked example. J Adv Nurs 2013;69:jan.12127.

57 Ambuehl J, Karrer A, Meer A, et al. Quality of life of survivors of paediatric intensive care. Swiss Med Wkly 2007;137:312-6.

58 Buysse CMP, Raat H, Hazelzet JA, et al. Surviving meningococcal septic shock: health consequences and quality of life in children and their parents up to 2 years after pediatric intensive care unit discharge. Crit Care Med 2008;36:596-602.

59 Knoester H, Bronner MB, Bos AP, et al. Quality of life in children three and nine months after discharge from a paediatric intensive care unit: a prospective cohort study. Health Qual Life Outcomes 2008;6:21.

60 Colville GA, Pierce CM. Children's self-reported quality of life after intensive care treatment. Pediatr Crit Care Med 2013;14:e85-92.

61 Namachivayam SP, Alexander J, Slater A, et al. Five-Year survival of children with chronic critical illness in Australia and New Zealand. Crit Care Med 2015;43:1978-85.

62 Polic B, Mestrovic J, Markic J, et al. Long-Term quality of life of patients treated in paediatric intensive care unit. Eur $J$ Pediatr 2013;172:85-90.

63 Choong K, Al-Harbi S, Siu K, et al. Functional recovery following critical illness in children: the "wee-cover" pilot study. Pediatr Crit Care Med 2015;16:310-8. 